\title{
Visualization of extracranial-intracranial bypass in moyamoya patients using intraoperative three-dimensional digital subtraction angiography with intravenous contrast injection and robotic C-arm: patient series
}

\author{
Naoki Kato, MD, ${ }^{1}$ Issei Kan, MD, ${ }^{1}$ Yukiko Abe, BS, ${ }^{2}$ Katharina Otani, PhD, ${ }^{3}$ Michihisa Narikiyo, MD,,${ }^{1,4}$ Gota Nagayama, MD, ${ }^{1}$ \\ Kengo Nishimura, MD, ${ }^{1}$ Ryosuke Mori, MD, ${ }^{1}$ Tomonobu Kodama, MD, ${ }^{1}$ Toshihiro Ishibashi, MD, ${ }^{1}$ and Yuichi Murayama, MD ${ }^{1}$ \\ 1'Department of Neurosurgery, The Jikei University School of Medicine Tokyo, Tokyo, Japan; ²Department of Radiology, The Jikei University Hospital, Tokyo, Japan; ${ }^{3}$ Siemens \\ Healthcare K.K., Advanced Therapies Innovation Department, Tokyo, Japan; and ${ }^{4}$ Department of Neurosurgery, Kawasaki Saiwai Hospital, Kanagawa, Japan
}

\begin{abstract}
BACKGROUND The authors describe a noninvasive intraoperative imaging strategy of three-dimensional (3D) digital subtraction angiography (DSA) with intravenous (IV) contrast injection, using indocyanine green (ICG) as a test bolus, during extracranial-intracranial (EC-IC) bypass surgery for moyamoya disease.

OBSERVATIONS Four patients underwent EC-IC bypass surgery in a hybrid operating room. During the surgery, bypass patency was verified using ICG videoangiography and Doppler ultrasonography. After skin closure, the patients under anesthesia underwent IV 3D-DSA with a robotic C-arm in which the scan delay time for the 3D-DSA scan was estimated from the arrival time of ICG during the ICG videoangiography. One day after the surgery, the patients underwent magnetic resonance angiography (MRA). The IV 3D-DSA images were retrospectively compared with those obtained with other modalities. Good bypass patency was confirmed on IV 3D-DSA, ICG videoangiography, Doppler ultrasonography, and postoperative MRA in all cases. The delay time determined using ICG videoangiography as a test bolus resulted in IV 3D-DSA with adequate image quality, allowing assessment of the spatial relationships between the vessels and anastomoses from all directions.
\end{abstract}

LESSONS To evaluate bypass patency and anatomical relationships immediately after EC-IC bypass surgery, IV 3D-DSA may be a useful modality. ICG videoangiography can be used to determine the scan delay time.

https://thejns.org/doi/abs/10.3171/CASE2057

KEYWORDS angiography; indocyanine green videoangiography; moyamoya disease; robotic C-arm; superficial temporal artery-middle cerebral artery bypass; three-dimensional digital subtraction angiography

Extracranial-intracranial (EC-IC) bypass surgery is one of the effective treatments for moyamoya disease..$^{1,2}$ The aim of this surgery is to restore cerebral vascularization through bypass. During the microsurgery, bypass patency can be evaluated using indocyanine green (ICG) videoangiography. ${ }^{3}$ However, the bypass can only be seen as a two-dimensional image from the outside and is limited by the field of view of the microscope before dural closure. Just after skin closure, intraarterial three-dimensional (3D) digital subtraction angiography (DSA) in a hybrid operating room (OR) is also one of the suggested modalities to confirm bypass patency. ${ }^{4,5}$ In addition, it allows examination of the anastomoses from different angles according to the flat panels' positions. However, this requires additional catheter insertion and thus increases the associated risks of thromboembolic complications and lengthens anesthesia time. Furthermore, catheterization may not be easy to include in a routine workflow. ${ }^{6}$ Recently, the feasibility of intraoperative contrast-enhanced imaging using a robotic $C$-arm system in the hybrid OR has been reported. ${ }^{7}$ Kan et al. have suggested that 3D-DSA with intravenous (IV) contrast medium administration performed with a robotic C-arm system could visualize intracranial aneurysms during clipping surgery as a potential alternative to computed tomography angiography

ABBREVIATIONS 3D = three-dimensional; CTA = computed tomography angiography; DSA = digital subtraction angiography; EC-IC = extracranial-intracranial; EDMS = encephalo-duro-myo-synangiosis; ICG = indocyanine green; IV = intravenous; $M C A=$ middle cerebral artery; $M R A=$ magnetic resonance angiography; OR = operating room; STA = superficial temporal artery; TIA = transient ischemic attack.

INCLUDE WHEN CITING Published January 25, 2021; DOI: 10.3171/CASE2057.

SUBMITTED November 2, 2020. ACCEPTED November 19, 2020.

(C) 2021 The authors, CC BY-NC-ND 4.0 (http://creativecommons.org/licenses/by-nc-nd/4.0/) 
(CTA). ${ }^{8}$ However, C-arm systems are not equipped with bolus tracking systems, ${ }^{9}$ and the adequate time for starting the scan after the start of the contrast medium injection is difficult to determine in a reliable way. ${ }^{10}$ In this case series, we used the delay time in intraoperative ICG videoangiography as the scan start time, that is, as a test bolus method, for intraoperative IV 3D-DSA after EC-IC bypass surgery. ${ }^{11}$ We compared the clinical findings of IV 3D-DSA with those obtained using intraoperative ICG videoangiography, Doppler ultrasonography, and postoperative magnetic resonance angiography (MRA).

\section{Study Description}

\section{Patient Population}

This study was approved by the institutional review board of the university hospital. Informed consent for bypass surgery and image acquisition using the robotic $\mathrm{C}$-arm system was obtained from all patients. We also obtained approval of data use regarding the treatment, images for research, and academic presentation from all the patients. For this case series, we reviewed four consecutive patients with symptomatic moyamoya disease who had been treated with EC-IC bypass surgery in a hybrid OR equipped with a robotic $\mathrm{C}$-arm system (ARTIS pheno, Siemens Healthcare $\mathrm{GmbH}$ ) and who had undergone 3D-DSA after IV administration of contrast medium at our institution between September 2018 and September 2020.

\section{EC-IC Bypass}

All patients underwent general anesthesia during the surgeries. The EC-IC bypass procedures were performed using microsurgical techniques under a microscope with integrated ICG videoangiography (Zeiss OPMI Pentero 900, Carl Zeiss Meditec; and MM80/SOH, Mitaka Kohki). ${ }^{12,13}$ The EC-IC bypass was created as an end-to-side anastomosis of the superficial temporal artery (STA) and middle cerebral artery (MCA) with 10-0 nylon strings. After creation of the anastomosis, the bypass patency was confirmed using both intraoperative ICG videoangiography and Doppler ultrasonography (DVM-4500, Hadeco). ${ }^{3,12}$ To determine the delay time for the subsequent IV 3D-DSA acquisition, we used ICG as a test bolus; i.e., we measured the time interval between the IV injection of ICG and arrival of the fluorescence in the anastomosed vessels in the monitor of the microscope.

\section{Imaging}

All IV 3D-DSA acquisitions were conducted using the robotic $\mathrm{C}$-arm system in the hybrid OR while the patients were still under anesthesia after surgery (Fig. 1A). A power injector (Medrad, Warrendale) was connected to the 20-gauge IV line, which was secured at the brachial vein for control of general anesthesia (Fig. 1B and C). The patients underwent 4-second DSA scanning, which consisted of a mask and fill run acquisition (tube voltage of 70 $\mathrm{kVp}$, dose of $0.36 \mu \mathrm{Gy} / \mathrm{Fr}$, and scan covering an angle of $200^{\circ}$ for 496 projections). The delay time was set as the arrival of fluorescence during intraoperative ICG videoangiography. The fill run was triggered immediately with the injection of undiluted contrast medium (loversol $320 \mathrm{mg} / \mathrm{mL}$, Optiray, Fuji Pharma; lohexol $300 \mathrm{mg} / \mathrm{mL}$, Iopaque, Fuji Pharma; or lomeprol $300 \mathrm{mg} / \mathrm{mL}$, Iomeron, BraccoEisai) at a rate of $4 \mathrm{~mL} / \mathrm{sec}$. The total injection time was the delay time and an additional 4 seconds for the 4-second DSA acquisition. The total contrast medium dose was $70-75 \mathrm{~mL}$. The acquired projection data were transferred to a commercial workstation (Syngo X Workplace, Siemens Healthcare $\mathrm{GmbH}$ ) for image reconstruction and postprocessing using the following parameters:

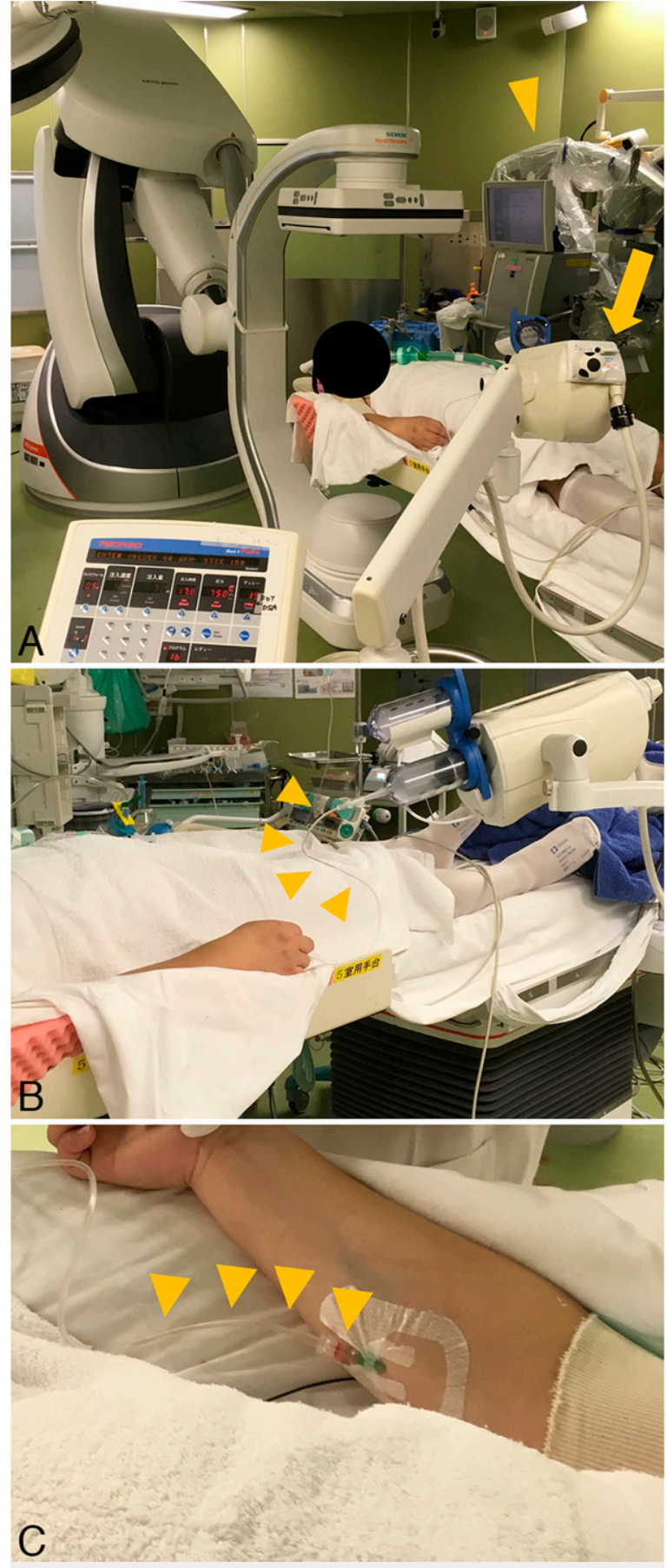

FIG. 1. Setup of IV 3D-DSA in a hybrid OR. A photograph showing the position of the robotic $\mathrm{C}$-arm, microscope (arrowhead), and injector of contrast medium (arrow) during image acquisition (A). A pressure-resistant tube (arrowheads) connected to the injector (B). The IV line (arrowheads) was secured at the vein of the right arm for the injection of contrast medium (C).

HU kernel type, smooth image characteristics, and a slice matrix of $256 \times 256.8$ 
TABLE 1. Characteristics of patients, treatment, contrast medium used, contrast medium dose, delay time, and radiation dose for IV 3D-DSA

\begin{tabular}{|c|c|c|c|c|c|c|c|}
\hline $\begin{array}{l}\text { Case } \\
\text { No. }\end{array}$ & Preop Symptom & Side & Treatment & Contrast Medium & $\begin{array}{l}\text { Contrast Medium } \\
\text { Dose }(\mathrm{mL})\end{array}$ & $\begin{array}{l}\text { Delay Time } \\
\text { (seconds) }\end{array}$ & $\begin{array}{l}\text { Radiation Dose } \\
\qquad(\mathrm{mGy})\end{array}$ \\
\hline 1 & TIA & $\mathrm{Lt}$ & Double STA-MCA bypass & $\begin{array}{l}\text { loversol, } 320 \mathrm{mg} \\
\text { iodine } / \mathrm{mL}\end{array}$ & 70 & 15 & 94.5 \\
\hline 2 & $\begin{array}{l}\text { Intracerebral } \\
\text { hemorrhage }\end{array}$ & $\mathrm{Rt}$ & $\begin{array}{c}\text { Single STA-MCA bypass } \\
\text { \& EDMS }\end{array}$ & $\begin{array}{l}\text { lohexol, } 300 \mathrm{mg} \\
\text { iodine } / \mathrm{mL}\end{array}$ & 70 & 10 & 83.9 \\
\hline 3 & TIA & $\mathrm{Lt}$ & $\begin{array}{c}\text { Single STA-MCA bypass } \\
\text { \& EDMS }\end{array}$ & $\begin{array}{l}\text { lomeprol, } 300 \mathrm{mg} \\
\quad \text { iodine } / \mathrm{mL}\end{array}$ & 70 & 18 & 100.6 \\
\hline 4 & Cerebral infarction & Lt & $\begin{array}{c}\text { Single STA-MCA bypass } \\
\text { \& EDMS }\end{array}$ & $\begin{array}{l}\text { lohexol, } 300 \mathrm{mg} \\
\text { iodine } / \mathrm{mL}\end{array}$ & 75 & 15 & 67.5 \\
\hline
\end{tabular}

MRA (MAGNETOM Skyra, Siemens Healthcare $\mathrm{GmbH}$ ) was routinely performed to verify bypass patency 1 day after the surgery. The 3D-MRA images were generated as maximum intensity projection images after time-of-flight acquisition using the following parameters: repetition time of $21 \mathrm{msec}$, echo time of $3.69 \mathrm{msec}$, flip angle of $18^{\circ}$, field of view of $180 \mathrm{~mm}$, slice matrix of $202 \times 320$, and acquisition time of 5.5 minutes.

\section{Evaluation of Bypass Imaging Compared With Other Modalities}

The patency of the bypass was retrospectively assessed on the IV 3D-DSA images, intraoperative ICG videoangiography, and postoperative MRA by a board-certified neurosurgeon (N.K., with 17 years of experience). The neurosurgeon examined the 3D-DSA images to find the inflow of contrast medium into the anastomosed STA using the rotate, zoom, and pan functions, while also changing the windowing, and compared the results of intraoperative ICG videoangiography and postoperative MRA in terms of whether the bypass graft was visualized. 3,14

The demographics of the patients and procedures are detailed in Table 1.

Good bypass patency was confirmed on IV robotic 3D-DSA, ICG videoangiography, and Doppler sonography in all four cases intraoperatively. The delay time estimated from intraoperative ICG videoangiography in each patient led to an adequate image quality of IV 3D-DSA for evaluating the inflow of contrast medium into the anastomosed vessels, even near bone structure, titanium plates for bone flap fixation, or metal staples used for closure of the scalp incision. The mean scan delay time was 14.5 seconds, and the image acquisition caused no side effects for the patients. Postoperative MRA verified graft patency in all four patients.

\section{Illustrative Cases}

\section{Case 1}

A double STA-MCA bypass of the left side was performed in a patient with moyamoya disease. The patient had been diagnosed with moyamoya disease after a transient ischemic attack (TIA) with symptoms in the left arm and was treated with an STA-MCA bypass of the right side (Fig. 2A). The intraoperative ICG videoangiography clearly showed the structures of both anastomosed recipient and donor vessels (Fig. 2B and C). After closure of the skin incision, the IV 3DDSA images were used to evaluate the bypass. The IV 3D-DSA clearly visualized the successful bypass patency through the osteoplastic craniotomy (Fig. 2D and E). Postoperative MRA on the following day confirmed the consistent flow of the anastomosed vessels (Fig. 2F).

\section{Case 2}

A patient with hemorrhagic unilateral moyamoya disease on the right side was treated with a single STA-MCA bypass combined with
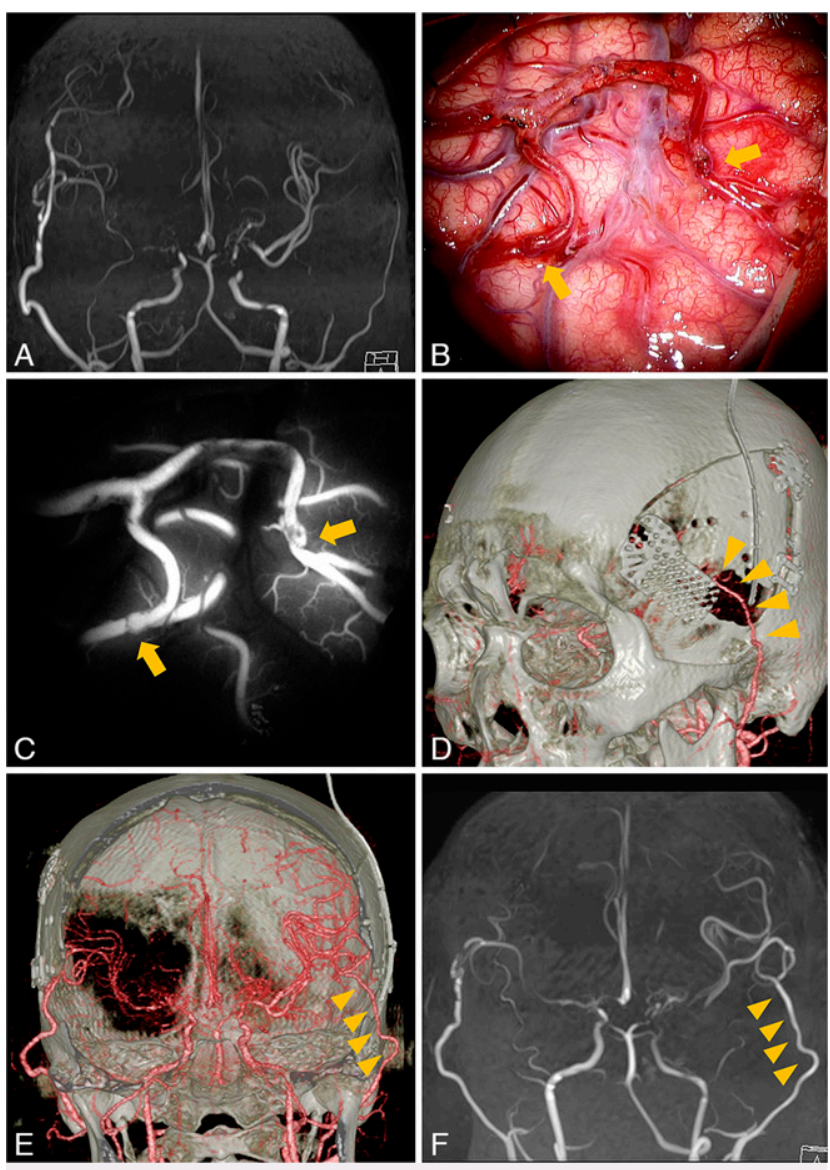

FIG. 2. Case 1. Preoperative MRA shows good bypass patency of the right side after initial treatment and stenotic major vessels of the untreated left side (A). Intraoperative image (B) and ICG videoangiography $(\mathbf{C})$ indicating structure of the anastomosed vessels (arrows). Successful graft patency (arrowheads) through the craniotomy verified by IV 3D-DSA, extracranial view (D) and coronal view (E). Postoperative MRA presents good bypass patency of the left side (F) (arrowheads). 
encephalo-duro-myo-synangiosis (EDMS) (Fig. 3A). Intraoperative ICG videoangiography showed good bypass patency (Fig. 3B and C). The inflow of contrast medium in the STA was visualized by IV 3D-DSA after skin closure (Fig. 3D and E). Patent anastomosis was verified by MRA on the day after the surgery (Fig. 3F).

\section{Case 3}

A patient with moyamoya disease diagnosed after a TIA was treated with a single STA-MCA bypass combined with EDMS on the left side (Fig. 4A). Intraoperative ICG videoangiography showed successful bypass patency (Fig. 4B and C). After closure of the skin incision, IV 3DDSA demonstrated good bypass patency through the craniotomy (Fig. 4D and E). Postoperative MRA on the following day confirmed the sufficient flow of the anastomosed vessels (Fig. 4F).

\section{Case 4}

A patient with moyamoya disease diagnosed after symptomatic cerebral infarction was treated with a single STA-MCA bypass combined with EDMS on the left side (Supplemental Fig. 1A). Successful anastomosis was confirmed by intraoperative ICG videoangiography
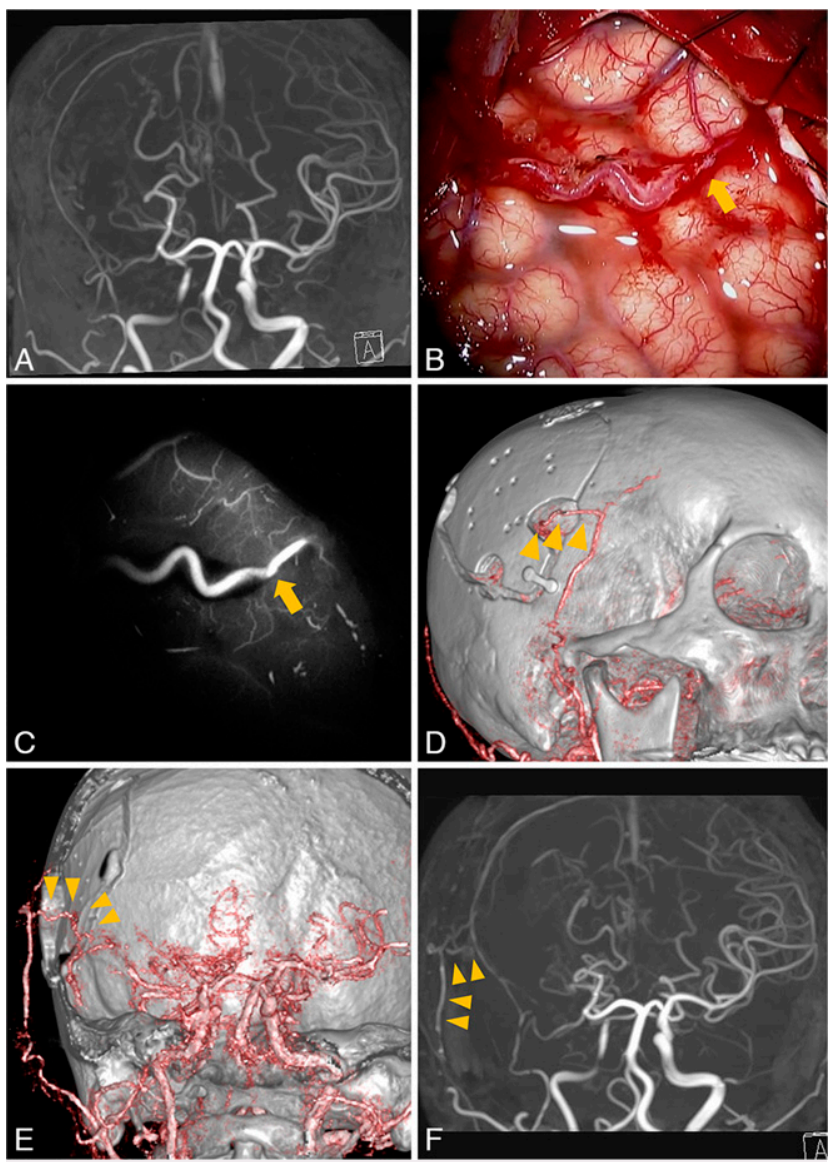

FIG. 3. Case 2. Preoperative MRA shows unilateral moyamoya disease of the right side (A). Intraoperative image (B) and ICG videoangiography (C) reveal good bypass patency (arrows). Graft patency (arrowheads) through the craniotomy confirmed by IV 3DDSA, extracranial view (D) and coronal view (E). Postoperative MRA shows successful bypass patency of the right side (F) (arrowheads).
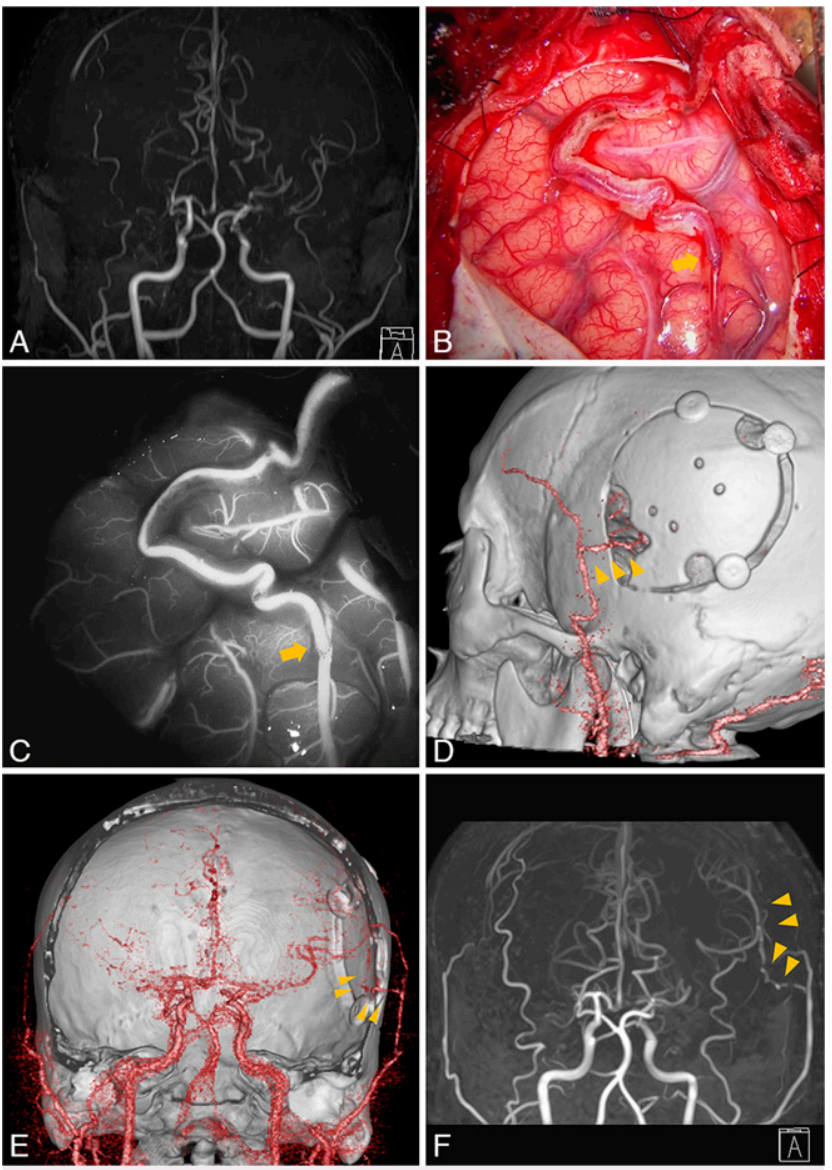

FIG. 4. Case 3. Preoperative MRA shows bilateral moyamoya disease (A). Intraoperative image (B) and ICG videoangiography (C) show good bypass patency (arrows). Graft patency (arrowheads) through the craniotomy confirmed by IV 3D-DSA, extracranial view (D) and coronal view (E). Postoperative MRA showed sufficient bypass patency of the left side (F) (arrowheads).

(Supplemental Fig. 1B and C). Subsequent IV 3D-DSA visualized adequate bypass patency (Supplemental Fig. 1D and E). Postoperative MRA on the following day verified the flow of the anastomosed vessels (Supplemental Fig. 1F).

\section{Discussion}

EC-IC bypass surgery has been established and widely used as a treatment modality for moyamoya disease. ${ }^{1}$ Because bypass occlusion during surgery could be experienced in some cases, it is important to assess the bypass patency intraoperatively. ${ }^{4}$ In this case series, we used IV 3D-DSA images acquired with an angiographic robotic C-arm system to evaluate the bypass patency during EC-IC bypass surgery and compared the results with those obtained using other modalities. Our workflow easily included IV 3D-DSA, which allowed us to verify successful anastomosis during the procedure immediately after closure of the skin incision.

Intraoperative ICG and fluorescein videoangiography are also established modalities to evaluate bypass patency; however, they have shortcomings. The bypass can only be evaluated on the surface from one direction and ICG videoangiography cannot be performed after closure of the skull. ${ }^{15}$ 


\section{Observations}

The image quality of IV 3D-DSA was sufficient for assessing the bypass patency, and the findings corresponded to those seen on ICG videoangiography and MRA. In addition, the spatial relationship of the bones, vessels, and anastomosis could be assessed from any angle on IV 3D-DSA by using the rotate, zoom, and pan functionalities of the workstation.

The IV 3D-DSA requires setting a scan delay time. ${ }^{8}$ We found that ICG videoangiography was useful for deciding the actual delay time, similar to a test bolus method, which can ensure that the scan is obtained during contrast enhancement. The delay time was measured using ICG videoangiography as the time interval between IV ICG injection and ICG arrival onto the brain surface. ${ }^{14,15}$ The actual time measurement was particularly important because the contrast bolus arrival time in the patient is affected by altered heart rate or blood pressure caused by anesthesia. ${ }^{11}$

Postoperative DSA is still known as the gold standard to verify the bypass function. Intraoperative DSA has also become a promising modality in the hybrid OR ${ }^{4,5,16,17}$ However, DSA is invasive because it requires a special catheter insertion into the investigated vessels. ${ }^{6}$ In contrast to catheter-directed DSA, IV 3D-DSA is noninvasive and requires only a few minutes until the availability of images. ${ }^{7,18}$ Hence, it has the advantage of decreasing the anesthesia time compared with intraoperative DSA and can be applied in routine workflow.

3D-CTA and MRA are noninvasive modalities that have diagnostic accuracy for assessing bypass patency comparable with that of DSA. ${ }^{19-23}$ One disadvantage of these modalities is that patient transfer to the examination room is necessary. In contrast, imaging in the hybrid OR has the advantage that revascularization can be confirmed intraoperatively while the patient is still located in the $\mathrm{OR}$. If compression or occlusion of the bypass graft related to closure of the skull and skin was detected, the failed bypass could be repaired immediately.7, 7,16

The drawback of this protocol was that the additional radiation dose and contrast medium dose to the patient appear to be similar to those for a CTA scan, but we believe that these are at an acceptable level for obtaining information regarding the bypass function and spatial relationships of vessels at a glance and preventing potential failed revascularization. Also, robotic $\mathrm{C}$-arms may not be available in every hybrid OR.

Our study had some limitations. Only a few cases from a single center were included, and studies with a larger sample size are needed to validate this method. This case series did not include negative controls, i.e., patients with failed bypass patency. A statistical analysis was not performed because of the small number of cases.

\section{Lessons}

The use of IV 3D-DSA using a robotic C-arm may be a useful noninvasive modality for confirming bypass patency immediately after the EC-IC bypass and could potentially become an alternative to intraoperative intraarterial 3D-DSA or postoperative CTA. The arrival time of ICG during videoangiography may be used as the delay time setting for IV 3D-DSA acquisition.

\section{References}

1. Czabanka M, Peña-Tapia P, Scharf J, et al. Characterization of direct and indirect cerebral revascularization for the treatment of European patients with moyamoya disease. Cerebrovasc Dis. 2011;32(4):361-369.

2. Miyamoto S, Yoshimoto T, Hashimoto N, et al. Effects of extracranial-intracranial bypass for patients with hemorrhagic moyamoya disease: results of the Japan Adult Moyamoya Trial. Stroke. 2014:45(5):1415-1421.

3. Woitzik J, Horn P, Vajkoczy P, et al. Intraoperative control of extracranial-intracranial bypass patency by near-infrared indocyanine green videoangiography. J Neurosurg. 2005;102(4): 692-698.

4. Yanaka K, Fujita K, Noguchi S, et al. Intraoperative angiographic assessment of graft patency during extracranial-intracranial bypass procedures. Neurol Med Chir (Tokyo). 2003;43(10):509-513.

5. Barrow DL, Boyer KL, Joseph GJ. Intraoperative angiography in the management of neurovascular disorders. Neurosurgery. 1992; 30(2):153-159.

6. Kaufmann TJ, Huston J 3rd, Mandrekar JN, et al. Complications of diagnostic cerebral angiography: evaluation of 19,826 consecutive patients. Radiology. 2007;243(3):812-819.

7. Mori R, Joki T, Matsuwaki Y, et al. Initial experience of real-time intraoperative $\mathrm{C}$-arm computed-tomography-guided navigation surgery for pituitary tumors. World Neurosurg. 2013;79(2): 319-326.

8. Kan I, Kato N, Otani K, et al. Intravenous 3-dimensional digital subtraction angiography during surgical treatment of intracranial aneurysm. World Neurosurg. 2019;126:533-536.

9. Shirasaka T, Hiwatashi A, Yamashita K, et al. Optimal scan timing for artery-vein separation at whole-brain CT angiography using a 320-row MDCT volume scanner. Br J Radiol. 2017;90(1070): 20160634

10. Struffert T, Deuerling-Zheng Y, Kloska S, et al. Flat detector CT in the evaluation of brain parenchyma, intracranial vasculature, and cerebral blood volume: a pilot study in patients with acute symptoms of cerebral ischemia. AJNR Am J Neuroradiol. 2010;31(8):1462-1469.

11. Henzler T, Meyer M, Reichert M, et al. Dual-energy CT angiography of the lungs: comparison of test bolus and bolus tracking techniques for the determination of scan delay. Eur J Radiol. 2012;81(1):132-138.

12. Charbel FT, Meglio G, Amin-Hanjani S. Superficial temporal artery-to-middle cerebral artery bypass. Neurosurgery. 2005; 56(1)(suppl):186-190.

13. Peña-Tapia PG, Kemmling A, Czabanka M, et al. Identification of the optimal cortical target point for extracranial-intracranial bypass surgery in patients with hemodynamic cerebrovascular insufficiency. J Neurosurg. 2008;108(4):655-661.

14. Prinz V, Hecht N, Kato N, et al. FLOW 800 allows visualization of hemodynamic changes after extracranial-to-intracranial bypass surgery but not assessment of quantitative perfusion or flow. Neurosurgery. 2014;10(suppl 2):231-239.

15. Narducci A, Onken J, Czabanka M, et al. Fluorescein videoangiography during extracranial-to-intracranial bypass surgery: preliminary results. Acta Neurochir (Wien). 2018;160(4):767-774.

16. Schaller K, Kotowski M, Pereira V, et al. From intraoperative angiography to advanced intraoperative imaging: the Geneva experience. Acta Neurochir Suppl (Wien). 2011;109:111-115.

17. Cho HH, Cheon JE, Kim SK, et al. Quantitative assessment of neovascularization after indirect bypass surgery: color-coded digital subtraction angiography in pediatric moyamoya disease. AJNR Am J Neuroradiol. 2016;37(5):932-938.

18. Carmody RF, Smith JR, Seeger JF, et al. Intracranial applications of digital intravenous subtraction angiography. Radiology. 1982; 144(3):529-534.

19. Besachio DA, Ziegler JI, Duncan TD, et al. Computed tomographic angiography in evaluation of superficial temporal to middle cerebral artery bypass. J Comput Assist Tomogr. 2010;34(3):437-439.

20. Kikuchi M, Asato M, Sugahara S, et al. Evaluation of surgically formed collateral circulation in moyamoya disease with 3D-CT angiography: comparison with MR angiography and X-ray angiography. Neuropediatrics. 1996;27(1):45-49. 
21. Langner S, Fleck S, Seipel R, et al. Perfusion CT scanning and CT angiography in the evaluation of extracranial-intracranial bypass grafts. J Neurosurg. 2011;114(4):978-983.

22. Chen $Q$, Qi $R$, Cheng X, et al. Assessment of extracranialintracranial bypass in moyamoya disease using 3T time-of-flight MR angiography: comparison with CT angiography. Vasa. 2014; 43(4):278-283.

23. Horn $P$, Vajkoczy P, Schmiedek $P$, et al. Evaluation of extracranialintracranial arterial bypass function with magnetic resonance angiography. Neuroradiology. 2004;46(9):723-729.

\section{Disclosures}

The Jikei University received grants from Siemens Healthcare K.K. for the current study as well as work outside the current study.

\section{Author Contributions}

Conception and design: Kato, Kan, Nishimura. Acquisition of data: Kato, Kan, Abe, Nagayama, Nishimura. Analysis and interpretation of data: Kato, Abe, Otani, Nishimura, Kodama. Drafting the article: Kato, Narikiyo, Nishimura, Murayama. Critically revising the article: Otani, Murayama. Reviewed submitted version of manuscript: Kan, Nagayama. Approved the final version of the manuscript on behalf of all authors: Kato. Statistical analysis: Otani. Administrative/technical/ material support: Kan, Mori, Ishibashi. Study supervision: Nishimura, Ishibashi, Murayama.

\section{Supplemental Information}

Online-Only Content

Supplemental material is available with the online version of the article. Supplemental Fig. 1. https://thejns.org/doi/suppl/10.3171/CASE2057.

\section{Correspondence}

Naoki Kato: The Jikei University School of Medicine Tokyo, Japan. nao-kth@jikei.ac.jp. 